\title{
Long range robust multi-terawatt MWIR and LWIR atmospheric light bullets
}

Jerome V. Moloney, Kolja Schuh, Paris Panagiotopoulos, M. Kolesik, S. W. Koch

Jerome V. Moloney, Kolja Schuh, Paris Panagiotopoulos, M. Kolesik, S. W. Koch, "Long range robust multi-terawatt MWIR and LWIR atmospheric light bullets," Proc. SPIE 10193, Ultrafast Bandgap Photonics II, 101930K (8 May 2017); doi: 10.1117/12.2262142

SPIE. Event: SPIE Defense + Security, 2017, Anaheim, California, United States 


\title{
Long Range Robust Multi-Terawatt
}

\section{MWIR and LWIR Atmospheric Light Bullets}

\author{
Jerome V Moloney ${ }^{\mathrm{a}, \mathrm{b}}$, Kolja Schuh ${ }^{\mathrm{a}}$, Paris Panagiotopoulos ${ }^{\mathrm{a}}$, M. Kolesik ${ }^{\mathrm{a}, \mathrm{b}}$ and S.W. Koch ${ }^{\mathrm{a}, \mathrm{c}}$ \\ ${ }^{\mathrm{a}}$ College of Optical Sciences, University of Arizona, Tucson 85721 ; ${ }^{\mathrm{b}}$ Arizona Center for \\ Mathematical Sciences, Department of Mathematics, University of Arizona, Tucson AZ 85721; \\ ${ }^{c}$ Department of Physics and Materials Science Center, Philipps-University, 35032 Marburg, \\ Germany
}

\begin{abstract}
There is a strong push worldwide to develop multi-Joule femtosecond duration laser pulses at wavelengths around 3.5-4 and $9-11 \mu \mathrm{m}$ within important atmospheric transmission windows. We have shown that pulses with a $4 \mu \mathrm{m}$ central wavelength are capable of delivering multi-TW powers at $\mathrm{km}$ range. This is in stark contrast to pulses at near-IR wavelengths which break up into hundreds of filaments with each carrying around $5 \mathrm{GW}$ of power per filament over meter distances. We will show that nonlinear envelope propagators fail to capture the true physics. Instead a new optical carrier shock singularity emerges that can act to limit peak intensities below the ionization threshold leading to low loss long range propagation. At LWIR wavelengths many-body correlations of weakly-ionized electrons further suppress the Kerr focusing nonlinearity around $10 \mu \mathrm{m}$ and enable whole beam self-trapping without filaments.
\end{abstract}

Keywords: long range propagation, filament, critical self-focusing, carrier shock, whole beam self-trapping.

\section{INTRODUCTION}

There are concerted efforts worldwide to develop midwave (MWIR) and longwave (LWIR) high energy ultrashort laser pulses (USPs) targeting the important 3-5 $\mu \mathrm{m}$ and 8-12 $\mu \mathrm{m}$ atmospheric transmission windows. Linear LWIR sources in the 8-12 $\mu \mathrm{m}$ transmission window are known to show enhanced propagation through fog and clouds relative to mid-IR and visible wavelengths. The potential usefulness of such sources for long range propagation is currently restricted by their short Rayleigh range and the consequent requirement for large aperture beam launch conditions. By packing energy into ultrashort laser pulses, the hope is that the intrinsic nonlinearity of air constituents could counteract strong diffraction effects and create remote filaments sustained over long paths. Currently available mid-IR 80 fs femtosecond pulsed sources at $3.9 \mu \mathrm{m}$ are limited to about $25 \mathrm{~mJ}$ in energy ${ }^{1}$ which is around half the predicted required energy to sustain a high-power single filament over multiple tens of meter ranges. In stark contrast to 800nm filaments which individually persist over a meter range, the latter should be sustainable over tens to hundreds of meters. Current USP sources in the important 8-12 $\mu \mathrm{m}$ window with sufficient energy to potentially create a filament in air are currently limited to $\mathrm{CO}_{2}$ gas lasers where recently 3 ps pulses with $3 \mathrm{~J}$ of energy have been reported ${ }^{2}$.

An important recent prediction is that the fundamental physics of USP propagation is significantly changed at longer wavelengths. In particular, the well-known critical self-focusing (collapse singularity) which leads to extreme local intensities at $800 \mathrm{~nm}^{3,4}$ while still evident, is no longer limited (regularized) via free electron plasma defocusing or intrinsic gas dispersion. Instead, it is predicted that an optical carrier shock wave develops early on, well before the collapse singularity is established and acts to limit the intensity growth by emitting recurrent bursts of dispersive waves that generate a broad featureless supercontinuum 5 . Ionization through tunneling now plays a relatively minor role and primarily acts to soften the extreme intensity spikes through additional weak plasma dispersion. Classical nonlinear envelope models fail here to capture the physics and the appropriate mathematical canonical description can now be expressed in terms of a full E.M field modified Kadomtsev-Petviashili equation ${ }^{6}$. The latter explicitly exhibits two 
singularities (1) the well-known 2D critical collapse and (2) an optical carrier shock wave where the shock velocity depends inversely on the local amplitude of the electric field.

The challenge to creating long-lived filaments with MWIR or LWIR USPs lies in the growth of the critical power as $\lambda^{2}$. Consequently the required power for self-focusing at $10 \mu \mathrm{m}$ in say, a 200fs pulse, is at least $300 \mathrm{~mJ}$, a factor of 100 larger than at $1 \mu \mathrm{m}$. Moreover, as we will show later, the highly detuned nature of the interaction at this long wavelength, brings the polarizability of the very weakly ionized, weakly interacting electrons associated with different ionized species in the gas into play. The important end result is an ultrafast dynamic negative lensing effect which acts nonuniformly in time to suppress the usual optical Kerr self-focusing and sustain a wide centimeter scale self-trapped beam capable of sustaining high power over multiple Rayleigh ranges. It should be remarked that shorter wavelength light bullets in the 3-4 $\mu \mathrm{m}$ and shorter IR wavelength range are primarily sustained by an energy reservoir that tends to persist on the order of the Rayleigh range of the initial launch beam. Previous claims to propagation over multiple Rayleigh ranges at $800 \mathrm{~nm}$ use the narrow $200 \mu \mathrm{m}$ filament diameter rather than the launch beam diameter. Finally, parallel recent advances in first principles quantum calculations of the optical nonlinear response of various inert gases now allows us minimize ad hoc phenomenological modeling of light-matter coupling and capture self-focusing, multiphoton or tunneling ionization within a single self-consist theory.

For the strongly off-resonant MWIR and LWIR excitation conditions, the light-matter coupling is in the regime of ultrafast adiabatic following where the optically induced polarization is slaved by the instantaneous values of the exciting light field. In the absence of dephasing effects, the occupation of the excited state follows adiabatically the exciting field, i.e. $f_{k} \propto E^{2}$. Thus, the system returns at the end of the excitation pulse into the ground state without any ionization. Clearly, strong-field ionization based on the interaction of single particles with the exciting field (multi photon ionization, above threshold ionization or tunnel ionization) can break this adiabatic following. However, these effects require very high intensities in order to be efficient.

An alternative way for the escape from adiabatic following is provided by many-body Coulomb interactions. In the field of optically excited semiconductors, it has been well established that the Coulombic scatterings of the optically induced microscopic polarizations can lead to dephasing. Since these processes depend on the degree of excitation in the system, these effects have been labeled as excitation induced dephasing (EID). Detailed experiment-theory comparisons have shown that these effects to dominate light-matter coupling under suitable conditions.

Even though the effects of EID are well-known in semiconductors, they are generally considered to be of lesser importance in atomic or molecular gases because these systems are typically rather dilute in comparison to solid-state densities. However, one should keep in mind that the decisive matrix element governing the strength of many-body scattering effects is given by the square of the active Coulomb interaction potential. We predict that these many-body Coulomb weakly correlated quasi-free electrons while evident at MWIR wavelengths, dominate the nonlinear response at LWIR wavelengths.

\section{THEORETICAL FOUNDATION}

The unidirectional pulse propagation equation (UPPE) model derived by us ${ }^{7,8}$, is essentially a unidirectional Maxwell propagator in that it fully resolves the underlying optical carrier wave. Despite this level of fine resolution, the resulting spectral solver shows almost ideal parallel scaling on our in-house SGI UV2000 machine enabling propagation of radial codes over multiple kilometers.

The spectral nature of the solver makes it possible to solve the linear propagation problem exactly. For a given medium permittivity $\epsilon(\omega)$, the linear propagation properties are encoded in the propagation constants of the modal fields. This allows one to incorporate the absorption and dispersion in the HITRAN database into the linear propagator thereby providing an enormous spectral coverage. While the dynamics driving mid-IR ultrashort pulse propagation in the atmosphere have already been studied extensively, the significance and/or interplay of multiple physical effects such as the delayed rotational Raman response, humidity levels, and avalanche ionization is as yet undetermined. In addition, the importance of many physical effects, as for example the percentage of water vapor in the form of humidity, depends strongly on the laser pulse wavelength and the material properties in the spectral regions of that wavelength but also in 
the regions located at 3rd, 5th, 7th harmonics and so on. The significance of the above physical effects may become even bigger as wavelength is increased beyond $4 \mu \mathrm{m}$.

The permittivity is in general complex-valued, so that not only the chromatic dispersive properties, but also absorption can be accounted for exactly. This is of great value in our case where the spectral window is enormous.

$$
k_{z}\left(\omega, k_{x}, k_{y}\right) \equiv \sqrt{\omega^{2} \epsilon(\omega) / c^{2}-k_{x}^{2}-k_{y}^{2}} .
$$

Medium properties other than linear enter the propagation model through the constitutive relations in Maxwell equations. The polarization $\vec{P}(x, y, z, t)$ and current density $\vec{J}(x, y, z, t)$ are given as functionals of the electric field $\vec{E}(x, y, z, t)$, thus the medium response is calculated from the history of the electric field $\vec{E}(x, y, z, t)$ independently at each spatial point.

The exact system of equations describing the evolution of modal amplitudes along the z-axis for the forward and backward propagating field is given below. Eq. 1 describes the forward propagating wave

$$
\partial_{z} \vec{E}_{k_{x}, k_{y},+}^{\perp}(\omega, z)=+i k_{z} \vec{E}_{k_{x}, k_{y},+}^{\perp}(\omega, z)+\sum_{s=1,2} \vec{e}_{s}^{\perp} \vec{e}_{s}^{\perp} \cdot\left[\frac{i \omega^{2}}{2 \epsilon_{0} c^{2} k_{z}} \vec{P}_{k_{x}, k_{y}}(\omega, z)-\frac{\omega}{2 \epsilon_{0} c^{2} k_{z}} \vec{J}_{k_{x}, k_{y}}(\omega, z)\right]
$$

and Eq. 2 describes the backward propagating wave.

$$
\partial_{z} \vec{E}_{k_{x}, k_{y},-}^{\perp}(\omega, z)=-i k_{z} \vec{E}_{k_{x}, k_{y},-}^{\perp}(\omega, z)-\sum_{s=1,2} \vec{e}_{s}^{\perp} \vec{e}_{s}^{\perp} \cdot\left[\frac{i \omega^{2}}{2 \epsilon_{0} c^{2} k_{z}} \vec{P}_{k_{x}, k_{y}}(\omega, z)-\frac{\omega}{2 \epsilon_{0} c^{2} k_{z}} \vec{J}_{k_{x}, k_{y}}(\omega, z)\right]
$$

Eq. (1) and Eq. (2) are the coupled unidirectional pulse propagation equations (UPPE) as described in detail in ref [7-8]. $\vec{e}_{1}$ and $\vec{e}_{2}$ are the unit vectors transverse to the direction of propagation.

The above UPPEs are mutually coupled through the medium response represented by the polarization and current density terms, and do require the knowledge of both the forward and backward propagating electric field. This is where the unidirectional approximation is made, i.e., that an accurately enough medium response can be calculated by the forward portion only:

$$
\vec{P}\left(\vec{E}_{-}+\vec{E}_{+}\right), \vec{J}\left(\vec{E}_{-}+\vec{E}_{+}\right) \rightarrow \vec{P}\left(\vec{E}_{+}\right), \vec{J}\left(\vec{E}_{+}\right)
$$

The unidirectional approximation is widely used in the modeling of pulse propagation in optics. The physical meaning and limitations of the "unidirectionality" assumption have been described in ref [9]. The canonical form of the scalar UPPE can be written in a simplified form as:

$$
\partial_{z} E_{k_{x}, k_{y}}(\omega, z)=i K\left(k_{x}, k_{y}, \omega\right) E_{k_{x}, k_{y}}(\omega, z)+i Q\left(k_{x}, k_{y}, \omega\right) P_{k_{x}, k_{y}}(\omega, z)
$$

with the exact linear propagator term

$$
K\left(k_{x}, k_{y}, \omega\right)=\sqrt{\omega^{2} \epsilon(\omega) / c^{2}-k_{x}^{2}-k_{y}^{2}}
$$

and the nonlinear response coupling term

$$
Q\left(k_{x}, k_{y}, \omega\right) \equiv \frac{\omega^{2}}{2 \epsilon_{0} c^{2} \sqrt{\omega^{2} \epsilon(\omega) / c^{2}-k_{x}^{2}-k_{y}^{2}}} .
$$

This scalar UPPE model has been shown to be remarkably robust ${ }^{9}$ against spontaneously generated backward generated waves. Additionally its relevance in describing MWIR and LWIR USP pulse propagation in condensed and gaseous media is best reflected by comparing it to the most sophisticated Nonlinear Envelope equation (NEE) model being widely used for propagation in the literature. The failure of NEE is illustrated in Figure 1 where there is a dramatic departure of UPPE and NEE after initial self-focusing has set in. The simulation is for a 100fs duration $3.9 \mu \mathrm{m}$ pulse in dry air.

The combination of optical carrier shock and critical self-focusing act in concert to limit the intensity growth in the UPPE case. Any nonlinear envelope model will start to blow-up and requires the artificial introduction of higher order 
Kerr (HOKE) terms to keep the peak intensity within physical bounds. There simply is no physics based justification to assume that NEE or any nonlinear envelope variant of NEE can properly capture the physics at these longer wavelengths.

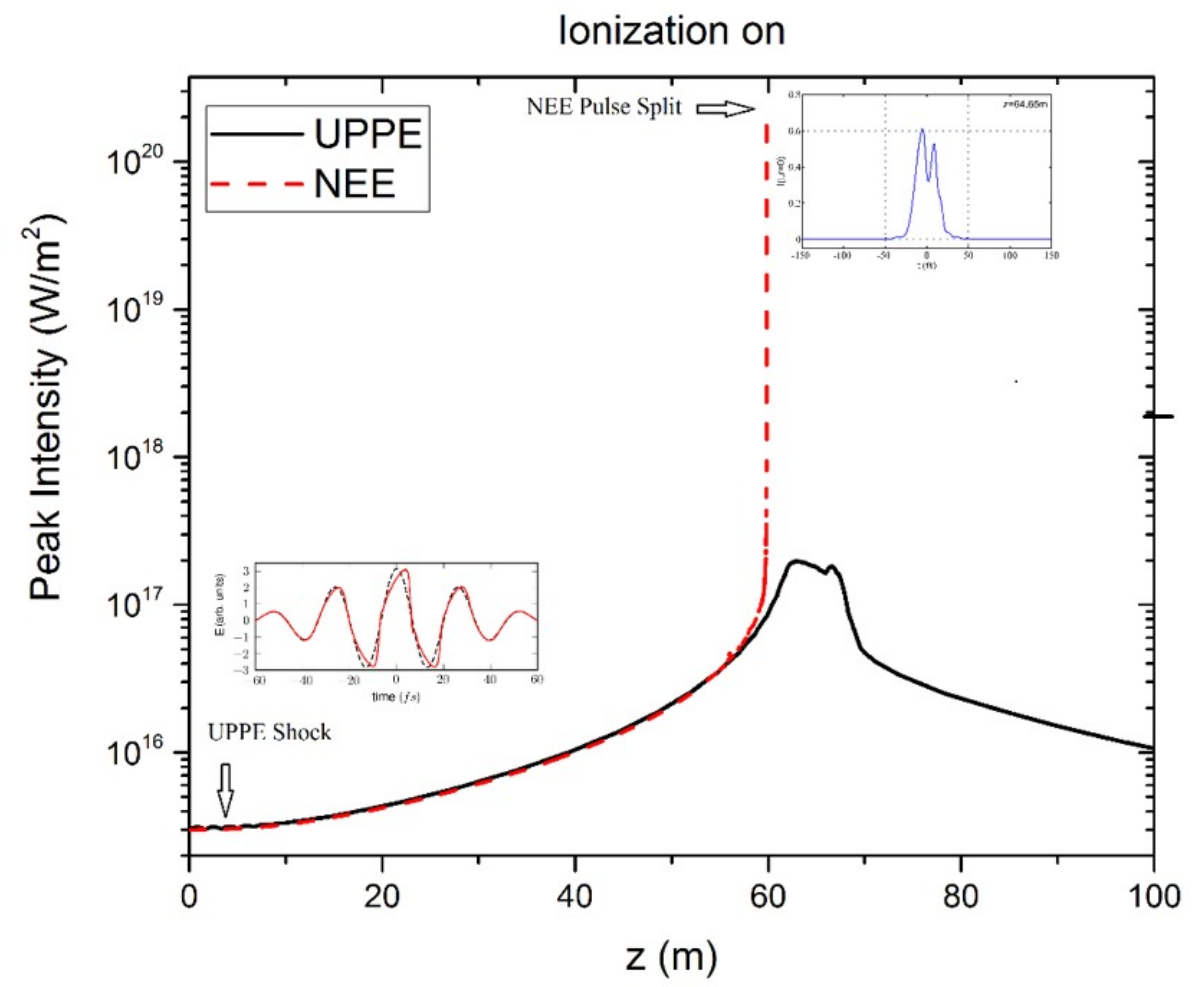

Figure 1 Comparison of the evolution of an identical initial $4 \mu \mathrm{m}$ pulse via the UPPE and the NEE. Lower inset on the left contrasts the strong optical carrier shock with a sinusoidal waveform after about 5 meters. Upper inset on the top right shows the eventual splitting of the NEE envelope due to weak GVD with the pulse dispersing subsequently.

Figure 2 shows a "phase diagram" reflecting the operative physics when the wavelength increases from the near-IR into the MWR and LWIR. The vertical intensity axis indicates achievable peak intensities in typical filaments. There are two regions that exhibit fundamentally different physical behavior and consequently greatly impact the propagation characteristics of USPs. At wavelengths below about $3.5 \mu \mathrm{m}$, we predict the classical critical self-focusing (critical collapse) behavior characteristic of $800 \mathrm{~nm}$ filaments. In this region shaded black in the figure, linear dispersion of the relevant species dominates over Kerr nonlinearity - In other words, the dispersion length is shorter is than the characteristic nonlinear length scale which depends on the strength of the Kerr coefficient. Here pulse propagation is captured by the classical Nonlinear Schrödinger equation (NLSE) and its many nonlinear envelope variants. In the lightly shaded region to the right which spans all wavelengths longer than about $3.5 \mu \mathrm{m}$, the physics changes fundamentally. Here, the characteristic nonlinear length scale is shorter than the dispersion length scale. Now a new singularity emerges in addition to the critical self-focusing collapse. The underlying optical carrier wave no longer remains sinusoidal and starts to self-steepen generating an E.M shock wave. 


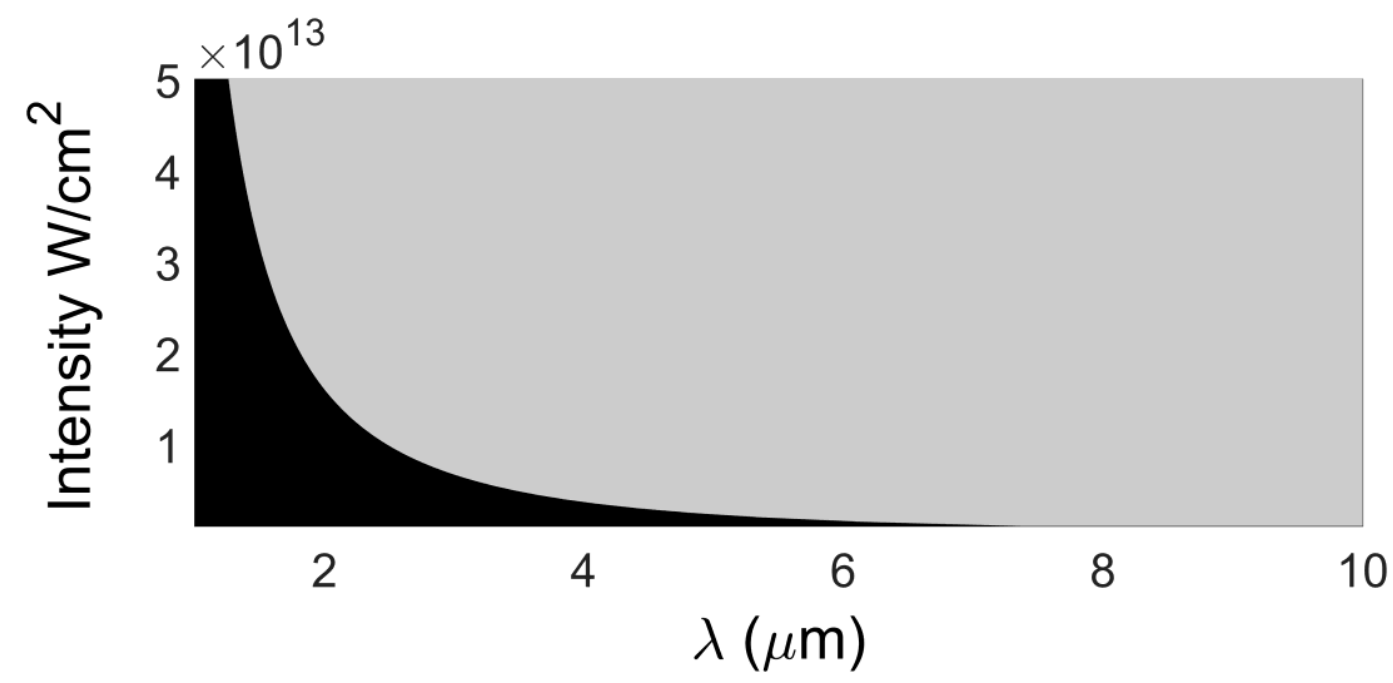

Figure 2. Phase diagram delineating the separation between nonlinear envelope (black) and UPPE(gray) behavior.

Figure 2 encapsulates the expected physical behavior and relevant propagation model needed as a function of varying wavelength. The vertical axis depicts typical peak intensity ranges achieved in a filament. The gray region includes optical carrier shock and critical self-focusing. The intensity increase due to self-focusing further accelerates the formation of the shock and the generation of broadband supercontinuum spectra. The shock disperses energies to shorter wavelengths thereby mediating the strong intensity growth.

For near-IR pulses $(800 \mathrm{~nm}, 1 \mu \mathrm{m}$ etc), dispersion dominates over nonlinearity and the appropriate physical and mathematical description of propagation of USPs is in terms of the well-known Nonlinear Schrödinger equation (NLSE) and its many variants (GNLSE, NEE etc). As the wavelength increases and transitions into the MWIR spectral region, the role of nonlinearity and dispersion are reversed. This is because the central pulse wavelength becomes further detuned from resonance whereas the optical nonlinearity remains essentially dispersionless. In this limit, the physics is now captured by the modified Kadomtsev-Petviashili $(\mathrm{mKP})^{6}$ equation, an equation that explicitly displays the coexistence of 2 singularities - an optical carrier shock and blow-up (critical self-focusing) singularity.

$$
\frac{\partial}{\partial \tau}\left(\frac{\partial E_{0}}{\partial z}+\frac{8 L_{0}}{L_{N L}} E_{0}^{2} \frac{\partial E_{0}}{\partial \tau}-\frac{L_{0}}{L_{\mathrm{DS}}} \frac{\partial^{3} E_{0}}{\partial \tau^{3}}\right)=\frac{L_{0}}{L_{\mathrm{DF}}} \nabla^{2} E_{0}
$$

In Eq. (4), $L_{\mathrm{NL}}, L_{\mathrm{DS}}$ and $L_{\mathrm{DF}}$ correspond to the characteristic lengths for the nonlinear Kerr effect, dispersion, and diffraction, which are defined by

$$
L_{\mathrm{NL}}=\frac{c}{n_{2} I_{0} \omega_{R}}, L_{\mathrm{DS}}=\frac{1}{4 a \omega_{R}^{3}}, L_{\mathrm{DF}}=\frac{k_{R} w_{0}^{2}}{2},
$$

where $n_{2}$ is the nonlinear refractive index of the material, $I_{0}$ is the initial pulse peak intensity, $\omega_{R}$ is a central frequency, and $w_{0}$ is the initial beam diameter at $1 / e^{2}$ radius. We define $L_{0}$ to be the shortest distance (usually $L_{\mathrm{NL}}$ or $L_{\mathrm{DF}}$ over which $E_{0}$ changes. The coefficient $a$ describes how the real part of the linear susceptibility deviates from a constant value. The operator $\nabla^{2}$ is the Laplacian.

A new twist appears at even longer wavelengths in the LWIR. Many-body microscopic correlations between weakly ionized electrons associated with different atoms/molecules in a gas can, through ionizations induced dephasing, further modify the effective Kerr lensing effect ${ }^{10,11}$ and lead to whole beam self-trapping rather than the creation of intense light filaments. This will be further discussed in section 4 . 
Finally, a key observation is that the critical power, free electron polarization and ionization (plasma) scale as $\lambda^{2}$.

$$
P_{\text {crit }}=\frac{0.3 \lambda^{2}}{n n_{2}} \quad P_{\mathrm{pl}}=-f(t) \frac{e^{2} \mu_{0}}{m} \lambda^{2} E \quad n_{\mathrm{pl}}=-f(t) \frac{e^{2} \mu_{0}}{2 m} \lambda^{2}
$$

where $f(t)$ is the electron density at time t. As we will show, while this scaling requires more power (and consequently energy) to reach critical, the resulting MWIR filaments and LWIR self-trapped beams are capable of delivering multiple TeraWatts of power (Joules of energy) over long ranges. This is in stark contrast to 800nm filaments whereby an individual filament transports about $5 \mathrm{GW}$ over distances less than a meter.

\section{CARRIER SHOCK MEDIATED MWIR FILAMENTS}

We now employ the scalar UPPE to simulate USPs of femtosecond duration at wavelengths in the vicinity of $4 \mu \mathrm{m}$. The actual wavelength here is not too significant as we do not include the HITRAN data and take a Kerr $n_{2}$ coefficient extrapolated from values at shorter wavelengths. Wahlstrand et al ${ }^{12}$ recently showed that the Kerr coefficient is essentially dispersionless over a broad spectral range. Consistent with the prediction of Fig. 2, we observe that a sequence of optical carrier shocks, accelerated by increasing intensity of the critical self-focusing process, is the main contributor towards limiting the rapid growth in intensity. The shock develops very early after just a few meters of propagation and is responsible for a sudden burst of emitted higher harmonic radiation. Multiple shock and broadband harmonic emission follow at irregular intervals over the full propagation path. This shock regularization typically limits the peak intensities such that ionization remains very weak. Consequently ionization (plasma) plays a minor role as an absorber of the propagating light. Ionization (plasma) dispersion does contribute significantly and contributes further to the background intrinsic dispersion in softening the self-steepening of the optical carrier wave.
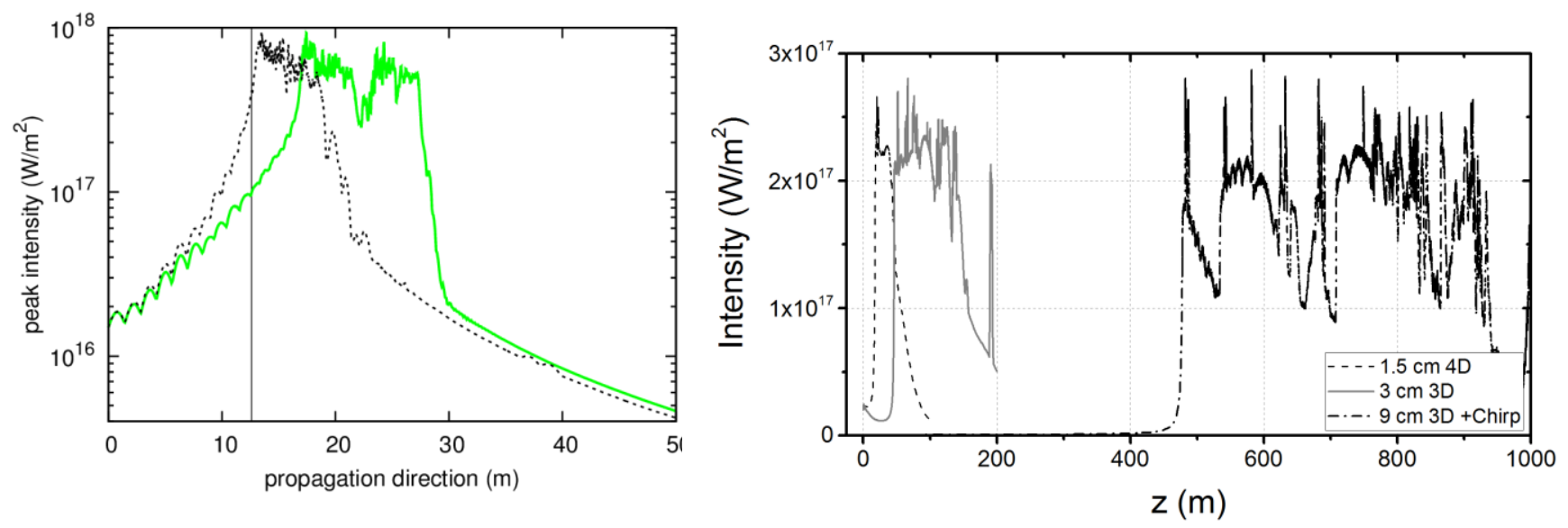

Figure 3. Left: Simulation of a $240 \mathrm{fs} 4 \mu \mathrm{m}$ pulse with a $.48 \mathrm{~cm}$ waist. Many-body effects delay the onset of filamentation but the behavior remains qualitatively the same. Right: Comparative simulations of a $24 \mathrm{fs} 1.5 \mathrm{~cm}$ and 3 $\mathrm{cm}$ and a $9 \mathrm{~cm}$ waist $350 \mathrm{fs} 4 \mu \mathrm{m}$ pulse. The $9 \mathrm{~cm}$ beam waist pulse is pre-chirped to create the onset of filamentation at $500 \mathrm{~m}$. The overall distance over which the filament is sustained is on the order of the Rayleigh range of the initial launched beam.

Figure 3 left frame compares a series of simulations of a $4 \mu \mathrm{m} 240 \mathrm{fs}$ pulse propagating in an Argon medium. The choice of Ar here is because the electronic Kerr coefficient of air is very close in magnitude to that of Argon and we avoid dealing with delayed Raman contributions arising from rovibrational transitions. The dashed curve utilizes the classical Kerr coefficient extrapolated from $800 \mathrm{~nm}$. The delayed solid curve shows that the influence of many-body Coulomb correlations of weakly ionized electrons from different atoms can reduce the effective Kerr self-focusing. This reduction 
is relatively minor here but becomes significant for LWIR pulses. The frame on the right of Fig. 3 shows how the Rayleigh range of the initial launch beam tends to dictates the range over which filamentation can be sustained. Increasing the beam waist extends the propagation path. Of course, a pre-chirp applied to the pulse can delay the onset of filamentation such that a remote filament can be created at ran

\section{MANY-BODY MEDIATED LWIR SELF-TRAPPED BEAMS}

Figure 4 below summarizes the significant impact of many-body correlations on the nonlinear optical response and consequently, on the delivery of high power (multiple TW) self-trapped high energy pulses at range. The left panel depicts the ionization degree with many-body contributions (solid curve) to the ionization degree at different peak pulse intensities. The schematic in the inset stresses that these Coluomb mediated correlations come from independent gas species. Full details of the theory and propagation simulations are contained in references [8-10]. The black arrow points to the maximum intensity reached within the self-trapped beam. This is significantly lower than the usual single species ionization threshold shown by the sharply increasing dashed curve. We chose Argon gas as our model as it has an electronic Kerr response comparable to that of air and avoids us having to deal with delayed Raman contributions of air that increase the overall Kerr response by a factor of $5^{13}$.
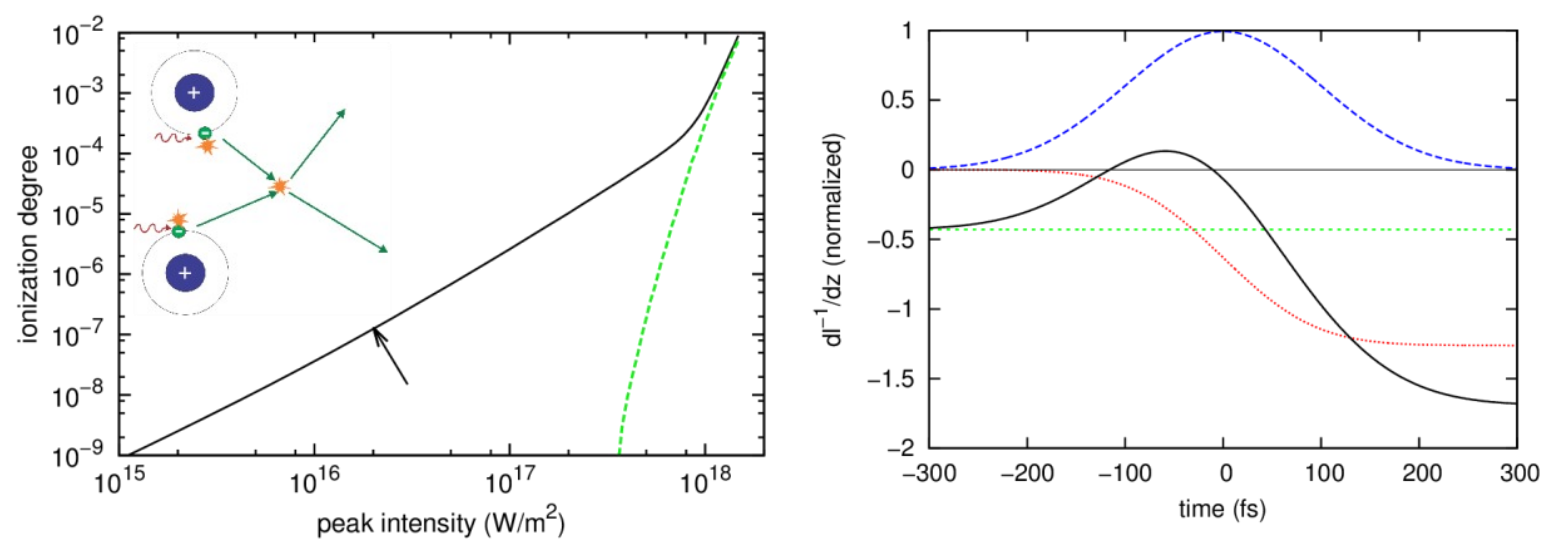

Figure 4. Left: Ionization degree of an Argon gas experiencing many-body effects during a 200fs pulse at $10 \mu \mathrm{m}$ computed by the microscopic quantum mechanical model (solid curve) and the single active electron quantum model (dashed curve) as function of pulse peak intensity. Right: Depiction of the Kerr contribution (dashed upper) towards the focusing and the many-body induced plasma contribution (lower dotted) as well as the constant diffraction contribution (horizontal dotted) towards the net focusing of a $10 \mu \mathrm{m}$ pulse with $2.4 \mathrm{TW}$ peak power and $1.2 \mathrm{~cm}$ width. Since the Kerr effect is proportional to the intensity, the dashed upper curve also tracks the shape of the pulse envelope. The combined effect (black solid) illustrates the net decrease of focusing in the central part of the pulse.

The strong suppression of the standard Kerr focusing at $10 \mu \mathrm{m}$ is clearly evident (black solid curve) from the right panel in Fig. 4. The standard electronic Kerr response (upper dashed curve) is offset by a combination of diffraction (horizontal dotted line) and by ionization (plasma) dynamic negative lensing (dotted negative curve). The latter is strongly dependent on pulse duration and peak intensity. Its impact at $4 \mu \mathrm{m}$, while evident in Fig. 3 (left panel), is much less than at $10 \mu \mathrm{m}$ due to the $\lambda^{2}$ scaling of the free electron polarization.

The impact of this suppressed positive lens on long range propagation is illustrated in Fig. 5 below. Here an initial 1 ps duration, $3.7 \mathrm{~cm}$ waist $10 \mu \mathrm{m}$ pulse is shown to propagate over a distance of $2 \mathrm{~km}$. An inspection of the transverse beam profile (not shown here) shows that the beam waist remains collimated starting from the initial launch point at $\mathrm{z}=0$ until it reaches a distance of $1500 \mathrm{~m}$. At this point the whole beam self-focuses gradually. Completely absent is the initial strong self-focusing associated with the onset of a filament [see Fig.3] Note that the Rayleigh range of the launched beam is about $310 \mathrm{~m}$ so that the pulse propagates on the order of 6-7 Rayleigh ranges. The linear propagation result is shown by the black solid line. It is important to stress that this is the first instance where it is predicted that a pulse can be launched over multiple Rayleigh ranges of the launch beam. 
We stress here that the optical carrier shocks still remain operative but the suppressed Kerr lensing tends to soften the shock behavior. Instead, the nonlinear lens suppression due to many-body Coulomb correlations tends to keep the beam from undergoing strong self-focusing. Instead, we now observe whole beam self-trapping in contrast to the shorter wavelength NIR or MWIR pulses which tend to create a relatively short range filament riding along on top of a sustaining wider beam (energy reservoir).

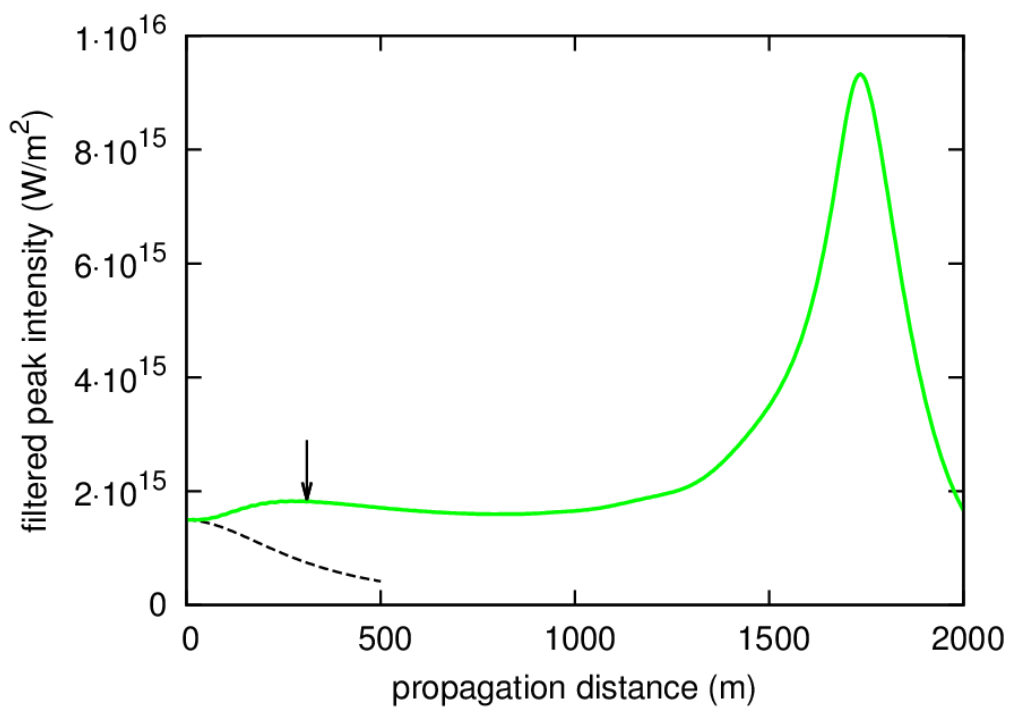

Figure 5. Evolution of the peak intensity of a picosecond duration, $3.7 \mathrm{~cm}$ wide beam waist pulse over $2 \mathrm{~km}$. The dashed curve the tracks the maximum intensity of a linearly diffracting beam. The black downward pointing arrow shows the Rayleigh range for this beam.

The results shown in Fig. 5 are particularly significant as they provide the first indicator that LWIR pulses can be launched in the atmosphere and propagate multiple Rayleigh ranges. The relatively weak ionization is in stark contrast to $800 \mathrm{~nm}$ filaments where ionization associate with individual filament intensity bursts acts as a significant loss channel via plasma absorption. By beating the diffraction limit, pulses can be launched with much smaller waists and possess all of the advantages such as the lesser influences of turbulence etc. Critically important to achieve long range is to launch the initial beam with a relatively flat phase front i.e collimated. Any pre-focusing in the optics of the laser can drive the effective lens from positive to negative without it being able to recover. In this situation, the beam spreading can be even faster than a Rayleigh range due to an added nonlinear defocusing.

\section{CONCLUSIONS}

In this article, we have presented new paradigms for multi-TW, multi-Joule USP propagation at range on the atmosphere. In stark contrast to NIR USPs, we find that MWIR USPs can still undergo filamentation but with filament waists on the order of a few millimeters. This enables one to pack more power and energy into a single filament. For LWIR pulses in the important $8-12 \mu \mathrm{m}$ atmospheric window, we predict that essentially infinite range, many-body Coulomb correlations between distant atoms/molecules in a gas can suppress a normally strong self-focusing lens. The end result is that a collimated initial pulse can propagate over multiple kilometers in the atmosphere. For both MWIR and LWIR pulses, the propagation is nearly lossless enabling such long propagation lengths. 


\section{REFERENCES}

[1] Mitrofanov A.V., , Sidorov-Biryukov D.A., Pugžlys A., Stepanov _. E.A., Andriukaitis G., Flöry T., Ališauskas S., Fedotov A.B., Baltuška A., Zheltikov A.M., "Mid-infrared laser filaments in the atmosphere", Nature Scientific Reports, 5, 8368 (2015)

[2] Haberberger, D and Tochitsky, S and Joshi, C, "Fifteen terawatt picosecond CO 2 laser system", Opt. Exp. 18, $17865-17875$.

[3] Mlejnek, M., Kolesik, M., Moloney, J.V. and Wright, E.M., "Optically turbulent femtosecond light guide in air.” Phys. Rev. Letts, 83 (15), pp. 2938-2941(1999).

[4] Couairon, A. and Mysyrowicz, A., Femtosecond filamentation in transparent media, Phys. Rep., 441, 47-189 (2007).

[5] Panagiotopoulos, P. and Whalen, P. and Kolesik, M. and Moloney, J.V.,Nature Photonics, 9, 543-548 (2015).

[6] Whalen, P., Panagiotopoulos, P., Kolesik, M. and Moloney J.V.,, "Extreme carrier shocking of intense longwavelength pulses," Phys. Rev. A 89, 023850 (2014)

[7] Kolesik M. and Moloney J.V., "Unidirectional pulse propagation equation", Phys. Rev. Letts.,89, 2839021 (2002).

[8] Kolesik, M. and Moloney,J.V, "Nonlinear optical pulse propagation simulation: From Maxwell's to unidirectional equations", Phys. Rev. E, 70, 036604 (2004).

[9] Kolesik, M., Jakobsen, P., and Moloney J.V., "Quantifying the limits of unidirectional ultrashort optical pulse propagation”, Phys. Rev. A, 86, 035801,(2012).

[10] Schuh, K and Moloney, JV and Koch, SW, "Interaction-induced nonlinear refractive-index reduction of gases in the midinfrared regime", Phys. Rev. E, 93, 013208 (2016) and references therein.

[11] Schuh, K., Kolesik, M., Wright, E.M., and Moloney, J.V., "Self-channeling of High-Power Long-Wave Infrared Pulses in Gases", Phys. Rev. Letts., 118, 063901 (2017).

[12] Wahlstrand, JK and Cheng, Y-H and Milchberg, HM, "Absolute measurement of the transient optical nonlinearity in $\mathrm{N}_{2}, \mathrm{O}_{2}, \mathrm{~N}_{2}, \mathrm{O}_{2}$, and Ar", Phys. Rev. A, 85, 043820, (2012).

[13] Pigeon, J.J., Tochitsky, S. Ya., Welch, E.C., and Joshi, C., "Measurements of the nonlinear refractive index of air, $\mathrm{N}_{2}$, and $\mathrm{O}_{2}$ at $10 \mu \mathrm{m}$ using four-wave mixing", Opt. Letts., 41, 3924 (2016). 